\title{
A New Modified Gant-Miwa-Thiersch Combined With Internal and External Rectal Sclerosant Injection Procedure for The Treatment of Full- Thickness Rectal Prolapse in Elderly Women
}

Jinxi Wang

Shanxi Bethune Hospital

Huiyu Li

Shanxi Bethune Hospital

Xiaoming Ma

Burn Treatment Center of Shanxi Province, Tisco General Hospital

Gang Du

Shanxi Bethune Hospital

Jun Ma

Shanxi Bethune Hospital

Xiaojing Ren

Shanxi Bethune Hospital

Fang Zhang

Shanxi Bethune Hospital

Xiushan Dong

Shanxi Bethune Hospital

Haoliang Zhao

Shanxi Bethune Hospital

Chongren Ren ( $\nabla$ renxiangxiang@126.com )

Shanxi Bethune Hospital

\section{Research Article}

Keywords: rectal prolapse, full-thickness rectal prolapse, Gant-Miwa-Thiersch procedure, nmGMTSI, recurrence

Posted Date: April 21st, 2021

DOI: https://doi.org/10.21203/rs.3.rs-419210/v1 
License: (c) (i) This work is licensed under a Creative Commons Attribution 4.0 International License. Read Full License 


\section{Abstract}

Background: Full-thickness rectal prolapse (FTRP) occurs frequently in in elderly women, and more than 100 surgical procedures have been proposed to restore FTRP. The Gant-Miwa-Thiersch (GMT) procedure is the most used treatment in China. However, the recurrence rates of GMT that reach as high as $23.8 \%$ is concerning. We described a new modified GMT combined with internal and external rectal sclerosant injection (nmGMTSI) procedure to address this problem.

Methods: The nmGMTSI was performed under spinal anesthesia in 34 frail, elderly female patients with FTRP. The surgical results of FTRP were assessed. Fecal incontinence and constipation were evaluated using the Wexner score, and anal canal rest pressure (ACRP), maximum anal systolic pressure (MASP), anorectal sensation thresholds (AST), and maximum rectal tolerance (MRT) using anorectal manometry preoperatively and postoperatively. The causes of recurrence and complications were analyzed.

Results: All patients were cured in accordance with clinical cure standard. The perioperative Wexner fecal incontinence score (WFIS) was $10.3 \pm 3.31$, which became $3.7 \pm 2.43(\mathrm{P}<0.0001)$ postoperatively. The perioperative ACRP was $2.0 \pm 0.56 \mathrm{kPa}$, which became $8.5 \pm 2.25 \mathrm{kPa}(\mathrm{P}<0.0001)$ postoperatively. The perioperative MASP was $4.5 \pm 1.16 \mathrm{kPa}$, which became $18.6 \pm 2.50 \mathrm{kPa}(\mathrm{P}<0.0001)$ postoperatively. However, there was no significant change between preoperative and postoperative Wexner constipation scores (WCS) (17.3 \pm 2.25 vs. $15.4 \pm 2.89, \mathrm{P}=0.1047)$. The AST were $38.1 \pm 5.34 \mathrm{~mL}$; after the operation, it became $23.5 \pm 3.61 \mathrm{~mL}(P=0.0002)$. The maximum rectal tolerance (MRT) was $157.1 \pm 16.73 \mathrm{~mL}$; after the operation, it became $121.2 \pm 12.45 \mathrm{~mL}(P=0.0009)$. The patients had no serious postoperative complications. The total relapse rate after nmGMTSI was $2.9 \%$ in the median 2 years follow-up period. The most common cause of relapse after nmGMTSI was the removal of infected threads used in the Thiersch procedure.

Conclusion: The benefits of nmGMTSI include low recurrence, complications, mortality, cost and wide adaptation, minimally invasive, technically simple. It should be considered as the first option for the treatment of FTRP in frail elderly women.

\section{Background}

Full-thickness rectal prolapse (FTRP) occurs frequently in older women [1]. Its chief clinical feature is a mass protruding from the anus following defecation. Occasionally, the symptoms of FTRP may occur spontaneously while coughing or weight-bearing. FTRP is a debilitating condition with a complex etiology, with obstetric trauma as the most common, iatrogenic sphincter injury during hemorrhoidectomy and fistula surgery, and external injury to the perineum [2].

More than 100 surgical procedures have been proposed to restore FTRP. The operative procedures for rectal prolapse can be broadly categorized as either abdominal or perineal approaches [3]. Traditionally, the perineal approach has been chosen for older, high-risk patients because of fewer surgical complications, shorter surgery time, and simpler anesthesia. Perineal procedures can be classified into 
two: to initiate fibrosis, such as submucosal sclerosant injection and to shorten the prolapsed rectum. These include Delorme's operation, Altemeier's operation, stapled transanal rectal resection (STARR), and Gant-Miwa-Thiersch (GMT). The GMT procedure is the most common treatment in China and Japan [45]. However, the recurrence rates of GMT are as high as $23.8 \%$ [4].

We described a new modified GMT combined with internal and external rectal sclerosant injection (nmGMTSI) procedure to address this problem. To the best of our knowledge, this is the first reported case of such an approach to treat FTRP, with significant results in frail elderly women.

\section{Methods}

The study subjects, including female patients clinically diagnosed with FTRP [6], were enrolled at the Colorectal Surgery Unit of Shanxi Bethune Hospital from January 2016 to December 2018. Patients were recruited based on the following criteria: age $>65$ years and those can't tolerate general anesthesia with chronic heart failure, chronic obstructive pulmonary disease, chronic renal failure requiring hemodialysis, liver cirrhosis or other conditions. Enterocele or cystocele was ruled out by bimanual examination. Whole blood cell analysis, blood clotting index, hepatic and renal function, and routine fecal tests were performed to rule out absolute contraindication of surgery. The clinical characteristics of eligible subjects are shown in Table 1. To explore the etiology of FTRP, risk factors of recruiter for FTRP were recorded in Table 2. All operations were performed by the same surgical team members of the unit. The surgical results of FTRP were assessed by clinical symptoms and bowel function. Bowel function of all patients in the study was measured for fecal incontinence and constipation using the Wexner score, anal canal rest pressure (ACRP), maximum anal systolic pressure (MASP), anorectal sensation thresholds (AST), and maximum rectal tolerance (MRT) by anorectal manometry before and 24 months after surgery. To analyze causes of recurrence and complications, hospital stay, operative time and intraoperative and postoperative complications were recorded in Table 3. Patients were followed-up every 3, 6, 12, and 24 months in the outpatient clinic. 
Table 1

Clinical characteristics of FTRP

\begin{tabular}{|ll|}
\hline & Total population $(\mathrm{n}=\mathbf{3 4})$ \\
\hline Age mean \pm SD $(\mathrm{yrs})$ & $76.2 \pm 9.14$ \\
\hline $\mathrm{BMI}\left(\mathrm{kg} / \mathrm{m}^{2}\right)$ mean $\pm \mathrm{SD}$ & $19.3 \pm 4.5$ \\
\hline Symptoms & \\
\hline Feeling of a bulge in the rectum during defecation & $29(85.3 \%)$ \\
\hline Feelings of prolapse without defecating & $1(14.7 \%)$ \\
\hline Diarrhea/Fecal incontinence & $26(76.4 \%)$ \\
\hline Constipation/obstructed defecation & $8(23.5 \%)$ \\
\hline Abdominal or anal pain & $8(24 \%)$ \\
\hline Blood discharge & $13(38.2 \%)$ \\
\hline Mucus discharge & $10(29.4 \%)$ \\
\hline
\end{tabular}

Table 2

Risk factors of FTRP

\begin{tabular}{|ll|}
\hline Factors & Total population $(\mathbf{n}=\mathbf{3 4})$ \\
\hline Vaginal delivery $\geq 3$ & $29(85.3 \%)$ \\
\hline$<3$ & $5(14.7 \%)$ \\
\hline Chronic obstructive pulmonary & $23(67.6 \%)$ \\
\hline Malnutrition /Long term vegetarians & $21(61.7 \%)$ \\
\hline Redundant rectosigmoid colon & $18(52.9 \%)$ \\
\hline Previous pelvic surgery & $13(38.2 \%)$ \\
\hline IBD or colitis & $12(35.3 \%)$ \\
\hline IBS & $8(23.5 \%)$ \\
\hline Solitary rectal ulcer & $5(14.7 \%)$ \\
\hline Family history of rectal prolapse & $4(11.8 \%)$ \\
\hline
\end{tabular}


Table 3

Surgical management, results, and complications

\begin{tabular}{|ll|}
\hline & Numbers \\
\hline Hospital stays (days) & $7.3 \pm 1.6$ \\
\hline Operative time (min) & $44 \pm 12$ \\
\hline FTRP curative ratio & $34 / 34(100 \%)$ \\
\hline Curative ratio of diarrhea or fecal incontinenc & $25 / 26(96.2 \%)$ \\
\hline Curative ratio of constipation & $4 / 8(50.0 \%)$ \\
\hline Recurrence of rectal prolapse & $1 / 34(2.9 \%)$ \\
\hline Rectal bleeding & 3 \\
\hline Ischaemic colitis & 2 \\
\hline Urinary retention & 2 \\
\hline Stenosis of the rectum & 1 \\
\hline Perianal abscess & 1 \\
\hline Infection of the thread used for the Thiersch procedure & 1 \\
\hline
\end{tabular}

\section{Operative procedures}

Surgery was performed under spinal anesthesia and antibiotics were used $0.5 \mathrm{~h}$ before the operation. The patient was placed in a lithotomy and Trendelenburg positions to prevent the small intestine from slipping into the Douglas pouch. The prolapsed rectum was pulled out of the anus completely using babcock forceps, and the anus was inserted using a rotating speculum anoscope. The rectal mucosa was transfixed by $2 / 0$ Vicryl thread at the 12,3,6, and 9 o'clock positions, $5 \mathrm{~cm}$ above the top of the prolapsed mucosa. The threads were not cut and pulled in four directions (Fig. 1a). Consecutive grasping of the prolapsed mucosa using hemostatic forceps was performed. The grips on the prolapsed rectal mucosa were ligated using a $3 / 0$ silk thread to create a tag in multiple transverse and longitudinal lines that were arranged at $0.5 \mathrm{~cm}$ intervals without excision of the tag (Fig. 1b). The sclerosant ( $1 \mathrm{~mL}, 50 \%$ XiaoZhiLing) was injected into the rectal submucosa proximal to the mucosal plication (Fig. 1c). The prolapsed rectal mucosa $1 \mathrm{~cm}$ above the dentate line was sutured with a previously uncut Vicryl thread 2/0 (Fig. 1d). The prolapsed rectum was completely restored by tightening the Vicryl thread (Fig. 1e). The index finger was placed into the rectum and the needle was inserted $8 \mathrm{~cm}$ from the $1.5 \mathrm{~cm}$ anal verge in the 3 and 9 o'clock positions. Under the guidance of the index finger and ultrasound, the sclerosant $(20 \mathrm{~mL} 100 \%$ XiaoZhiLing) was injected into the pelvic rectum space (Fig. 1f,g). Thereafter, four 3-mm-long vertical incisions were made in the midline of the anal verge anteriorly and posteriorly at the 12, 3, 6, and 9 o'clock 
positions. All the incisions were sutured using a 0 Vicryl thread to encircle the entire anal verge. The suture was tied over an index finger (Fig. 1h).

\section{Statistical analyses}

The data were evaluated using the Student's t-test with SPSS 22.0 (SPSS Inc., Chicago, Illinois, USA) and were expressed as mean $\pm S D$. Differences were considered statistically significant at $P<0.05$.

\section{Results}

Between January 2016 and December 2018, a total of 34 female patients with FTRP underwent nmGMTSI. The clinical characteristics of the patients are listed in Table 1. The median age was $76.2 \pm$ 9.14 years (range 65-87 years), while the BMI was $17.3 \pm 4.5 \mathrm{~kg} / \mathrm{m}^{2}$ (range 12-23) with most patients underweight. All patients complained of a mass protruding through the anus. In $85.3 \%$ of patients, the mass sometimes protruded; in $14.7 \%$ of patients, the mass protruded all times. The initial condition in most patients was diarrhea or fecal incontinence (76.4\%), while constipation was the initial presentation in $23.6 \%$ of patients. Some patients presented with abdominal or anal pain, blood discharge, and mucus discharge (Table 1). The potential risk factors analyzed were multiparity, chronic obstructive pulmonary disease, malnutrition, redundant rectosigmoid colon, previous pelvic surgery, colitis, and IBS (Table 2). This result was slightly different from that in Western societies.

The average hospitalization time was $7.3 \pm 1.6$ days (range $5-9$ ) and most of the patients were discharged before one week. The median operative time was $44 \pm 12 \mathrm{~min}$ (range, 30-60 min). The symptoms of FTRP disappeared completely after surgery in all patients (Table 3 ). All patients were cured in accordance with clinical cure standard (Fig. 1i) [6]. Twenty-six patients suffered from preoperative diarrhea or fecal incontinence, which improved in 25 patients after surgery (Table 3 ). The perioperative Wexner fecal incontinence score (WFIS) was 10.3 \pm 3.31 , which became $3.7 \pm 2.43(P<0.0001)$ postoperatively. The ACRP was $2.0 \pm 0.56 \mathrm{kPa}$; after the operation, it became $8.5 \pm 2.25 \mathrm{kPa}(\mathrm{P}<0.0001)$. The MASP was $4.5 \pm 1.16 \mathrm{kPa}$, after the operation it became $18.6 \pm 2.50 \mathrm{kPa}(\mathrm{P}<0.0001)$ (Fig. 2a). Eight patients had preoperative constipation, which improved in four patients after surgery. However, there was no significant change between preoperative and postoperative Wexner constipation scores (WCS) (17.3 \pm 2.25 vs. $15.4 \pm 2.89, P=0.1047)$. The AST were $38.1 \pm 5.34 \mathrm{~mL}$, after the operation it became $23.5 \pm 3.61$ $\mathrm{mL}(\mathrm{P}=0.0002)$. The MRT was $157.1 \pm 16.73 \mathrm{~mL}$; after the operation, it became $121.2 \pm 12.45 \mathrm{~mL}(\mathrm{P}=$ 0.0009). (Fig. 2b).

Three patients (8.8\%) had rectal bleeding and were controlled using an anal pack and conservative measures. Two patients $(5.9 \%)$ showed urinary retention after surgery and were controlled by indwelling catheters for one week. Two patients $(5.9 \%)$ showed ischemic colitis and were controlled by conservative measures. One patient (2.9\%) had stenosis of the rectum six months after the operation and was controlled by dilatation of the anus with a metal anoscope. Lastly, one patient (2.9\%) showed perianal abscessat 12 months after the operation, which was due to infection of the thread used for the Thiersch 
procedure. Anti-infective therapy was ineffective and rectal prolapse recurred one year after the infected thread had been removed (Table 3).

\section{Discussion}

Rectal prolapse (RP) is not fatal but it is a severe problem that can seriously influence the quality of life of patients and most commonly affects older women. There are two types of RP. Type I, which is also called mucosal prolapse, involves the protrusion of the mucosa and is usually less than $3 \mathrm{~cm}$ long. Type II, also called full-thickness rectal prolapse (FTRP), involves full-thickness extrusion of the rectal wall characterized by concentric folds in the prolapsed mucosa [6]. This study focused on the treatment of FTRP in older women.

Numerous operative approaches have been described for the treatment of FTRP. The various approaches can be classified into two groups: transabdominal and transperineal [3]. The choice of the initial treatment was based on the assessment, age, comorbidities, and grading of prolapse. Transperineal surgery, which is traditionally reserved for patients who are deemed unsuitable for abdominal operation (the frail and elderly) and those who do not tolerate general anesthesia, consists of Delorme's, Altemeier's, STARR, and GMT operations. Delorme's procedure involves mucosal and submucosal dissection, plication of the remaining muscle layer, and mucosal anastomosis. Delorme's procedure has been reported to cause significant bleeding and perforation after surgery [7]. Altemeier's procedure involves excision of the redundant rectum or sigmoid colon. It is more appropriate for obstructive defecation syndrome [8]. It has a risk for fatal complications, such as anastomotic breakdown, rectal bleeding, and perianal abscess. This approach has been reported to have a postoperative mortality rate of $1.6 \%$ after surgery [9-10]. The STARR operation involves excision of the redundant rectal mucosa with a colorectal anastomosis that only applies to a mucosal protrusion of less than $5 \mathrm{~cm}$ long [11-12]. Since FTRP is a benign disease, the operative method should be simple, has wide adaptation, and has low postoperative mortality rate and cost.

One surgical technique for FTRP is the Gant-Miwa-Thiersch procedure (GMT) (mucosal plication with anal encircling). It can be used irrespective of the length of the rectum and colon prolapse [4]. lida et al. reported that there were no postoperative complications out of 166 patients who underwent GMT. GMT is not popular in Europe and the United States, while it plays a major role in the treatment of rectal prolapse in China and Japan [4-5]. Possible reasons include anatomical and dietary factors. In Western societies, an elongated sigmoid colon is commonly observed in elderly institutionalized patients with chronic constipation. A redundant, elongated sigmoid colon is prone to rectal prolapse [13]. In China, the pathophysiology of FTRP is usually related to several anatomic concerns, such as obstetric trauma causing weakness of pelvic floor muscles associated with connective tissue, MMP- 1 involved in chronic obstructive pulmonary disease that leads to loosely attached rectal mucosa to the underlying muscularis [14] (Table 2), and malnutrition and aging resulting in loss of the normal sacral curvature [15-16]. Due in part to long-term vegetarians in China, constipation seldom occurs [17]. Diarrhea or fecal incontinence was thought to be the common accompanying symptoms of FTRP (Table 1). Clinical results of GMT 
showed improved defecation with minimal complications [18]. Therefore, selecting surgical approaches should consider the exact causative factors and anatomical variations and tailored according to the patient's disease characteristics. However, the recurrence rate after GMT was $23.8 \%$ within a maximum follow-up period of 14 years [4]. We modified the GMT and combined it with square transfixion with submucosal and ischiorectal space injection sclerotherapy to address this problem.

Compared to GMT,we made the following three improvements in nmGMTSI. First, the grip of the full thickness of the rectal wall was transfixed by $2 / 0$ Vicryl thread at $12,3,6$, and 9 o'clock positions, $5 \mathrm{~cm}$ above the top of the prolapsed mucosa. Similar to GMT, multiple tags were created. After the rectal mucosa, the tissue $1 \mathrm{~cm}$ above the dentate line was sutured with the previously uncut Vicryl thread 2/0. The prolapsed rectum was completely restored by tightening the Vicryl thread. This improvement can significantly shorten the operation time and prevent recurrence in the short term after surgery, in which inflammatory adhesions have not yet formed. Second, longitudinal injection of the sclerosant in each of the four quadrants of the rectal submucosal area promotes inflammatory response and scarring, which prevents long-term mucosal prolapse recurrence. Finally, the sclerosant was injected into the pelvic rectal space. This improvement was mainly due to the weakness of the pelvic floor muscles associated with connective tissue. The sclerosant initiates an inflammatory reaction resulting in fibrosis outside the rectal wall and the perirectal tissue that leads to the wall of the rectum adheres to the perirectal tissue, preventing recurrence of prolapse of the rectal wall. Of course, sclerosant should be avoided in the anal sphincter.

Evaluation of the nmGMTSI depends on the results and complications of the surgery. In this study, the WFIS was significantly lower after surgery (Fig. 2a). This study matches the study of Yamana et al. [18], as this study found improvement in incontinence in more than $96.2 \%$ of patients (Table 3 ). One possible explanation is that the ACRP and MASP were significantly increased after anal encircling and the wall of the rectum adhered to the perirectal tissue (Fig. 2a). Constipation was partially improved in this study (Table 3). The AST and MRT were downregulated compared to preoperative values. But the WCS was no significantly different between the preoperative and postoperative (Fig. 2b). One possible reason is that scar formation in the rectal submucosal area would result in outlet obstruction. As shown in Table 3 , some patients had complications such as bleeding, colitis, and stenosis, but all recovered satisfactorily with conservative therapies. It is worth noting that the thread used for anal encircling can be infected, and its removal was usually necessary. In such cases, recurrence tends to occur after removal.

In our study, the overall recurrence rate after nmGMTSI was $2.9 \%$ during a period of two years with no operative death. The nmGMTSI is effective for diarrhea or fecal incontinence. There are, of course, some limitations in this research. Postoperative follow-up time was not long enough. The nmGMTSI is less effective for constipation or obstructed defecation. The score on patient's satisfaction score are not validated.

\section{Conclusion}


The nmGMTSI has a series of benefits, including wide adaptation; minimally invasive; technically simple; and with low cost, complications, mortality, and recurrence. Hence, we recommend this procedure be used as the first choice for FTRP in frail elderly women with pelvic floor muscle weakness.

\section{Abbreviations}

FTRP: Full-thickness rectal prolapse; STARR: stapled transanal rectal resection; GMT: Gant-MiwaThiersch; nmGMTSI: new modified GMT combined with internal and external rectal sclerosant injection; WFIS: Wexner fecal incontinence score; ACRP: anal canal rest pressure; MASP: maximum anal systolic pressure; WCS: Wexner constipation scores; AST: anorectal sensation thresholds; MRT: maximum rectal tolerance; IBD: inflammatory Bowel Disease; IBS: irritable bowel syndrome.

\section{Declarations}

\section{Acknowledgements}

Not Applicable

\section{Funding}

This study was funded by the "136 medical developing Project" of Shanxi Province, P. R. China.

\section{Availability of data and materials}

All data generated or analysed during this study are available from the corresponding author on reasonable request.

\section{Authors' contribution}

Jinxi Wang and Huiyu Li wrote the manuscript. Xiaoming Ma, Gang Du, and Jun Ma performed all operations. Xiaojing Ren and Fang Zhang did all the analysis and drew figures. Xiushan Dong, Haoliang Zhao and Chongren Ren designed the study and revised the manuscript. All authors read and approved the final manuscript.

\section{Ethics approval and consent to participate}

The study was authorized by the ethics committee of Shanxi Medical University (approval number:YXLL2016-008). The study methodology conformed to the standards set by the Declaration of Helsinki. Each patient was informed of the contingent risks of the study and provided written informed consent.

\section{Consent for publication}

All authors approved the final manuscript for publication. Written informed consent was obtained from the patients and/or their legal guardians for publication, and any accompanying images, sex, age of 
these patients.

\section{Competing interests}

The authors declare no potential conflicts of financial interests.

\section{References}

1. Gourgiotis S, Baratsis S. Rectal prolapse. Int J Colorectal Dis. 2007; 22(3):231-43.

2. Kumar N, Kumar D. Fecal incontinence and rectal prolapse. Indian J Gastroenterol. 2019; 38(6):465469.

3. Hrabe J, Gurland B. Optimizing Treatment for Rectal Prolapse. Clin Colon Rectal Surg. 2016; 29(3):271-6.

4. lida Y, Honda K, Saitou H, Munemoto Y, Tanaka H. Modified Gant-Miwa-Thiersch procedure (mucosal plication with anal encircling) for rectal prolapse. Colorectal Dis. 2019; 21(5):588-594.

5. Shen Z, Yang GG, Zhang XF, Qiu JM, Yu YY. Clinical analysis of 31 cases with rectal prolapse undergoing modified Gant-Miwa procedure and anal encircling. Zhonghua Wei Chang Wai Ke Za Zhi. 2013; 16(7):641-4.

6. van der Schans EM, Paulides TJC, Wijffels NA, Consten ECJ. Management of patients with rectal prolapse: the 2017 Dutch guidelines. Tech Coloproctol. 2018; 22(8):589-596.

7. Marchal F, Bresler L, Ayav A, Zarnegar R, Brunaud L, Duchamp C, Boissel P. Long-term results of Delorme's procedure and Orr-Loygue rectopexy to treat complete rectal prolapse. Dis Colon Rectum. 2005; 48(9):1785-90.

8. Trompetto M, Tutino R, Realis Luc A, Novelli E, Gallo G, Clerico G. Altemeier's procedure for complete rectal prolapse; outcome and function in 43 consecutive female patients. BMC Surg. 2019; 19(1):1.

9. Pinheiro LV, Leal RF, Coy CS, Fagundes JJ, Martinez CA, Ayrizono Mde L. Long-term outcome of perineal rectosigmoidectomy for rectal prolapse. Int J Surg. 2016; 32:78-82.

10. Kim M, Reibetanz J, Schlegel N, Krajinovic K, Köstler H, Germer CT, Isbert C. Recurrence after perineal rectosigmoidectomy: when and why? Colorectal Dis. 2014; 16(11):920-4.

11. Picciariello A, Papagni V, Martines G, De Fazio M, Digennaro R, Altomare DF. Post-operative clinical, manometric, and defecographic findings in patients undergoing unsuccessful STARR operation for obstructed defecation. Int J Colorectal Dis. 2019; 34(5):837-842.

12. Zanella S, Spirch S, Scarpa M, Ricci F, Lumachi F. Long-term outcome of stapled transanal rectal resection (STARR) versus stapled hemorrhoidopexys (STH) for grade III-IV hemorrhoids: preliminary results. In Vivo. 2014; 28(6):1171-4.

13. Sun $C$, Hull T, Ozuner G. Risk factors and clinical characteristics of rectal prolapse in young patients. J Visc Surg. 2014; 151(6):425-9.

14. Cao LL, Yu J, Yang ZL, Qiao X, Ye H, Xi CL, Zhou QC, Hu CC, Zhao CJ, Gong ZL. MMP-1/TIMP-1 expressions in rectal submucosa of females with obstructed defecation syndrome associated with 
internal rectal prolapse. Histol Histopathol. 2019; 34(3):265-274.

15. Kim du H, Sung DH, Min YK. Hypophosphatemic osteomalacia induced by low-dose adefovir therapy: focus on manifestations in the skeletal system and literature review. J Bone Miner Metab. 2013; $31(2): 240-6$.

16. Zhu Z, Xu L, Zhu F, Jiang L, Wang Z, Liu Z, Qian BP, Qiu Y. Sagittal alignment of spine and pelvis in asymptomatic adults: norms in Chinese populations. Spine (Phila Pa 1976). 2014; 39(1):E1-6.

17. Liu Y, Gao X, Ding Y, Zhou Y, Liu X, Wang H, Wang Q, Ma B, Yao S. Effectiveness and safety of light vegetarian diet on functional constipation with gastrointestinal damp-heat pattern: An exploratory study protocol for randomized controlled trial. Medicine (Baltimore). 2019; 98(50):e18325.

18. Yamana T, Iwadare J. Mucosal plication (Gant-Miwa procedure) with anal encircling for rectal prolapse-a review of the Japanese experience. Dis Colon Rectum. 2003; 46(10 Suppl):S94-9.

\section{Figures}



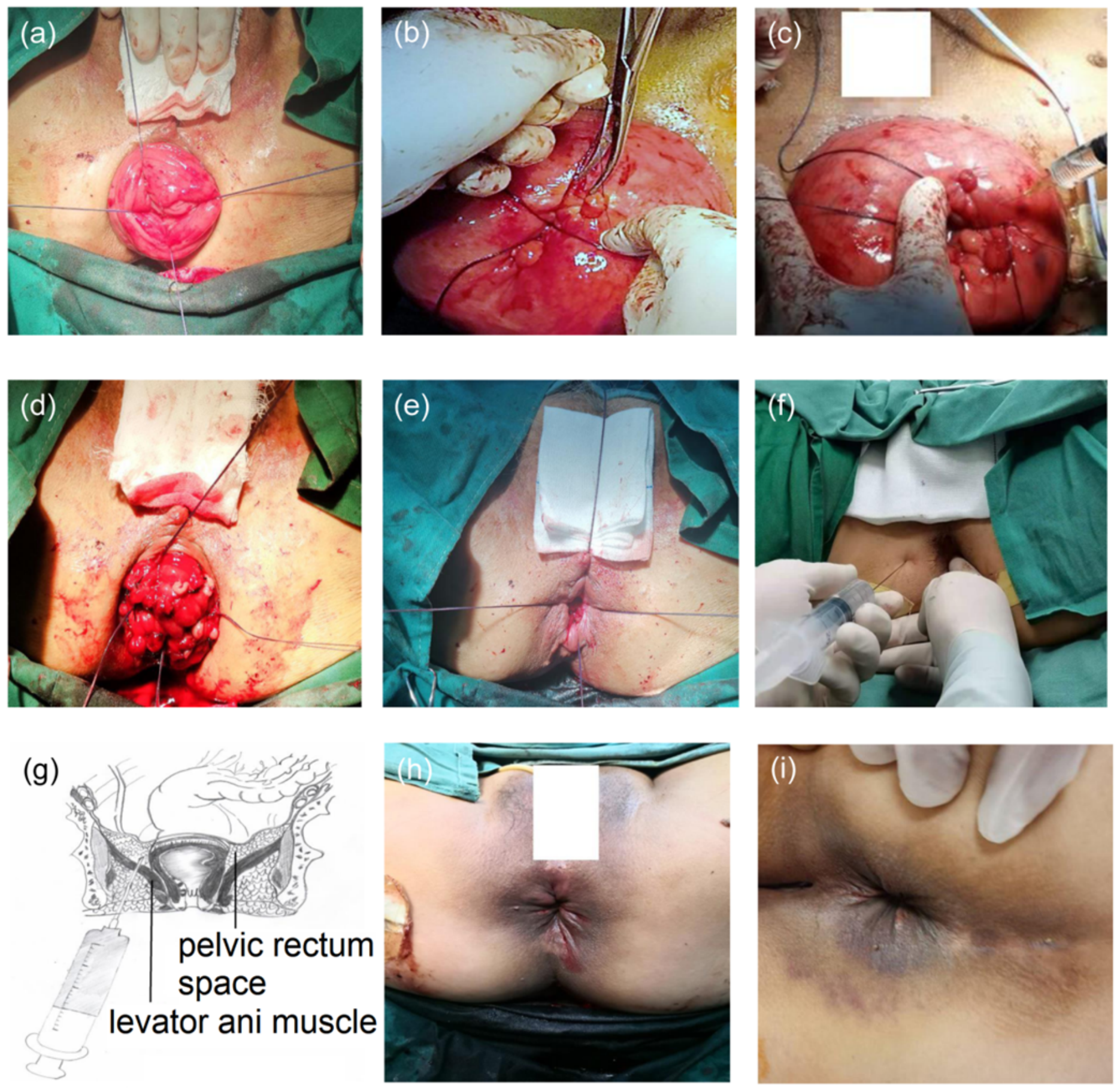

\section{Figure 1}

Operative procedure for nmGMTSI. (a) The prolapsed rectum was pulled out of the anus completely. Grips of the rectal mucosa were transfixed by $2 / 0$ Vicryl thread at 12,3,6, and 9 o'clock position, $5 \mathrm{~cm}$ above the top of the prolapsed mucosa. (b) Grips of rectal prolapsed mucosa was transfixed by silk thread. (c) Sclerosant was injected in rectal submucosa proximal to mucosal plication. (d) The prolapsed rectal mucosa $1 \mathrm{~cm}$ above the dentate line was sutured with the previous uncut Vicryl thread 2/0. (e) The prolapsed rectum was restored completely by tightening the Vicryl thread. (f) Sclerosant was injected in 
pelvic rectum space. $(\mathrm{g})$ Anatomic drawings of the injection in pelvic rectum space. (h) The Thiersch procedure was performed. (i) The results in clinical follow-up 3 months after surgery.

(a)
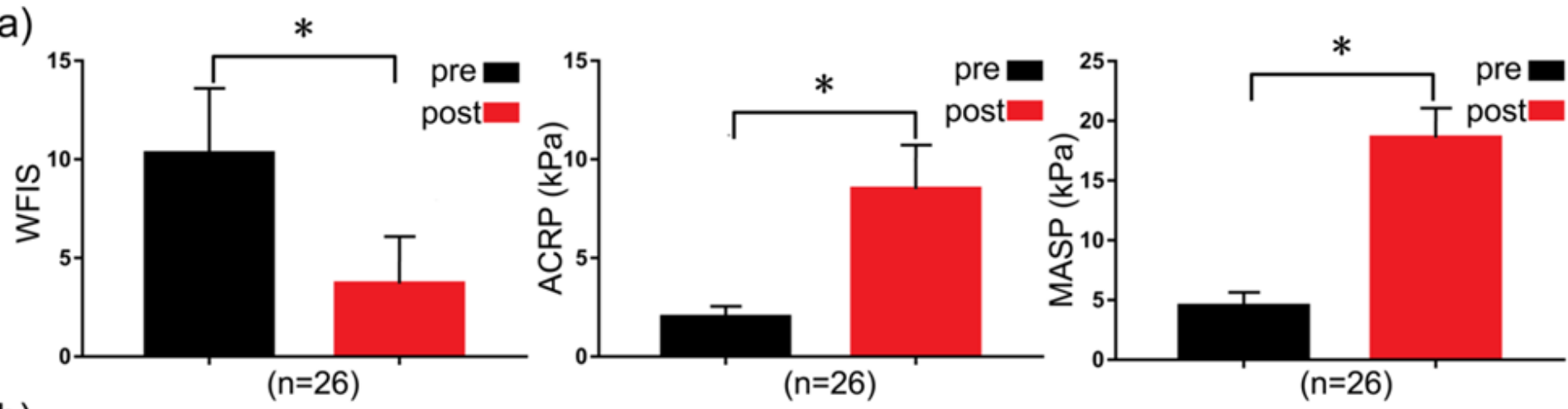

(b)
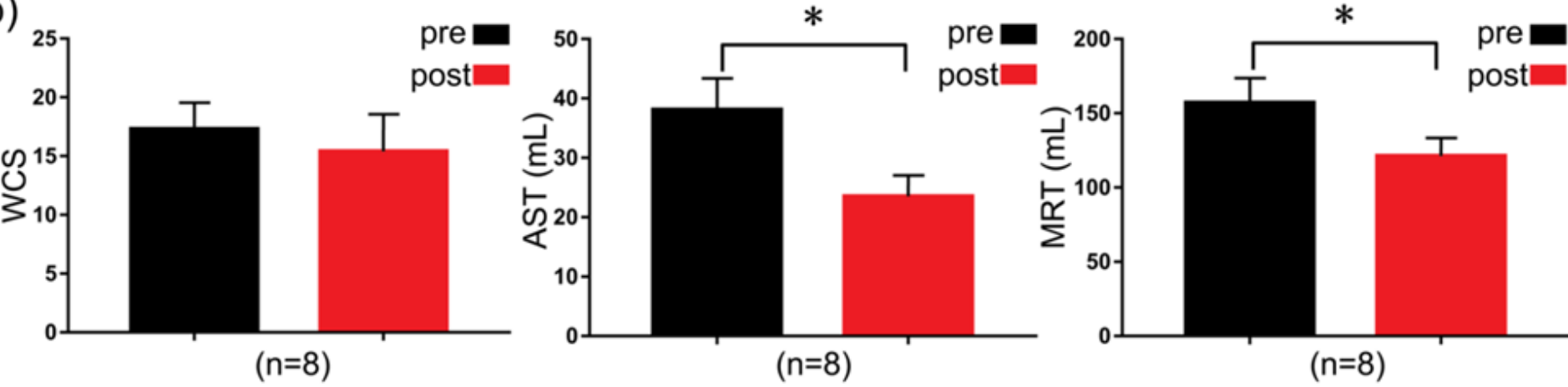

\section{Figure 2}

Surgical results of nmGMTSI. (a) Improvement of incontinence after nmGMTSI. WFIS, ACRP and MASP were evaluated for patients preoperatively and postoperatively. Quantitative data (mean \pm SD) were shown; ${ }^{*} \mathrm{P}<0.05$, Student's t-test. pre: preoperative, post: postoperative. (b) Partial improvement of constipation incontinence after nmGMTSI. WCS, AST and MRT were evaluated for patients preoperatively and postoperatively. 